Advanced i nst r ument at i on of f requency modul at i on AFM for subnanomet er-scal e 2D/3D measur ement $s$ at sol i d- I i qui d i nt erfaces

\begin{tabular}{|c|c|}
\hline 著者 & Fukuma Takeshi \\
\hline 著者別表示 & 福間 岡川士 \\
\hline $\begin{array}{l}\text { j our nal or } \\
\text { publ i cat i on titl e }\end{array}$ & NanoSci ence and Technol ogy \\
\hline vol une & 97 \\
\hline page $r$ ange & 435-460 \\
\hline year & 2015-01- 01 \\
\hline URL & ht t p: //doi . or g/10. 24517/00009133 \\
\hline
\end{tabular}




\title{
Advanced Instrumentation of Frequency Modulation AFM for Subnanometer-scale 2D/3D Measurements at Solid-Liquid Interfaces
}

Takeshi Fukuma

\begin{abstract}
Since the first demonstration of true atomic-resolution imaging by frequency modulation atomic force microscopy (FM-AFM) in liquid, the method has been used for imaging subnanometer-scale structures of various materials including minerals, biological systems and other organic molecules. Rencetly, there have been further advancements in the FM-AFM instrumentation. Three-dimensional (3D) force measurement techniques are proposed for visualizing 3D hydration structures formed at a solid-liquid interface. These methods further enabled to visualize 3D distributions of flexible surface structures at interfaces between soft materials and water. Furthermore, the fundamental performance such as force sensitivity and operation speed have been significantly improved using a small cantilever and highspeed phase detector. These technical advancements enabled direct visualization of atomic-scale interfacial phenomena at $1 \mathrm{frame} / \mathrm{sec}$. In this chapter, these recent advancements in the FM-AFM instrumentation and their applications to the studies on various interfacial phenomena are presented.
\end{abstract}

\section{Introduction}

In 1991, frequency modulation atomic force microscopy (FM-AFM) was invented by Albrecht et al. for operating dynamic-mode AFM in vacuum [1]. In vacuum, a $Q$ factor of a cantilever resonance $(Q)$ is much higher than that in air. While the higher $\mathrm{Q}$ factor provides a better minimum detectable force $\left(F_{\min }\right)$, it results in a narrower measurement bandwidth $(B)$ in amplitude modulation AFM (AM-AFM). FM-AFM is free from this problem, as $B$ is independent of $Q$.

In 1995, Giessibl et al. presented the first true atomic-resolution images obtained by FM-AFM in ultrahigh vacuum (UHV) environment [2]. Since then, FM-AFM

Takeshi Fukuma

Division of Electrical Engineering and Computer Science, Kanazawa University, Kakuma-machi, 920-1192 Kanazawa, Japan, e-mail: fukuma@staff.kanazawa-u.ac.jp 
has been widely used for atomic- or molecular-scale studies on conducting, semiconducting and insulating materials [3]. Until 2005, the method was used mostly in UHV while only few studies were performed in liquid $[4-10]$ or air $[6,11]$. This is partially because many researchers considered that the high $\mathrm{Q}$ factor in vacuum is essential for the high performance of FM-AFM and hence found no merits in liquid-environment operation. In addition, early studies of the liquid-environment FM-AFM revealed a number of technical difficulties in operating FM-AFM in liquid wtih sufficient stability and resolution [4-8].

In 2005, Fukuma et al. presented the first true atomic-resolution images obtained by FM-AFM in liquid [12]. This breakthrough was achieved by the following three technical improvements. First, they used a relatively stiff cantilever (spring constant: $k>20 \mathrm{~N} / \mathrm{m}$ ) for suppressing instabilities such as cantilever thermal vibration and adhesion events known as jump-to-contact. Secondly, they used a small cantilever oscillation amplitude (peak-to-zero amplitude: $A<0.5 \mathrm{~nm}$ ) for enhancing the sensitivity to short-range interaction force. Finally, they developed a low-noise cantilever deflection sensor to achieve thermal-noise-limited performance with a stiff cantilever and a small oscillation amplitude [13].

Since 2005, FM-AFM has been used for subnanometer-scale imaging of various materials in liquid. Examples of the early applications include minerals $[12,14-16]$ and biological systems $[14,17-22]$. While these results highlighted the high spatial resolution and wide applicability of liquid-environment FM-AFM, they also clarified its fundamental limitation. At solid-liquid interfaces, all the interactions are mediated by solvent molecules (typically water). The solvent molecules have non-uniform three-dimensional (3D) distribution. Thus, the two-dimensional (2D) information obtained by FM-AFM is often insufficient for understanding the mechanisms of solid-liquid interfacial phenomena.

In 2010, Fukuma et al. presented the first subnanometer-resolution imaging of 3D force distribution at a solid-liquid interface [23]. In the method, the tip is scanned vertically as well as laterally to cover the whole $3 \mathrm{D}$ interfacial space. During the scan, the frequency shift $(\Delta f)$ of the cantilever resonance is recorded to produce a 3D $\Delta f$ image. The obtained 3D $\Delta f$ image can be converted to a 3D force image using Sader-Jarvis equation [24]. As the force applied to the tip should be strongly influenced by the local distribution of solvent molecules, the subnanometer-scale contrasts observed in a 3D force image are often attributed to the 3D solvation structures. This argument has been further supported by the agreement between the experimentally obtained 3D force images of mica-water interface and water density distributions calculated by simulation [23, 25].

Since 2010, FM-AFM has been used for visualizing vertical-2D or 3D force distributions at various solid-liquid interfaces [23,25-35]. For interfaces between water and a rigid inorganic crystal, atomistic molecular dynamics (MD) simulation studies have been started towards detailed understanding of the imaging mechanism [?, 36-40]. In the meanwhile, 3D measurements at interfaces between water and soft materials such as lipid bilayers [26] suggested another possibility of this technique. At a soft interface, not only solvent molecules but also surface structures are thermally fluctuating. Thus, 3D force distribution images should reflect 
3D distributions of water and flexible surface structures. While this may add another complexity in the interpretation of the obtained 3D data, the capability of direct 3D imaging of soft interfacial structures can make significant advancements in molecular-scale understanding of various interfacial phenomena.

As described above, the application techniques of liquid-environment FM-AFM have been making rapid progress. However, the fundamental AFM performance such as force sensitivity and operation speed has remained almost unchanged. Recently, this has started to limit the application range of this technique. For example, insufficient $B$ of FM-AFM often prevents imaging dynamic behaviors of interfacial phenomena. Insufficient $F_{\text {min }}$ often prevents increasing pixel resolution in 3D force measurements and studying inhomogeneous interfacial structures. To overcome these limitations, we have recently made some improvements in the fundamental performance.

In the first part of this chapter, we describe recent progress in the instrumentation of liquid-environment FM-AFM. We explain the basic principle of our 3D force measurement technique referred to as 3D scanning force microscopy (3D-SFM). We also present the technical development that we made for the improvement of the fundamental performance such as force sensitivity and operation speed. In the second part of this chapter, we present high-resolution 2D and 3D FM-AFM imaging of various materials including minerals, organic molecules and biological systems. We show subnanometer-scale 2D imaging of lipid-cholesterol complexes, $\mathrm{CaF}_{2}(111)$ surfaces and tubulin protofilaments. We also present the results of subnanometerscale 3D imaging of mica-water and lipid-water interfaces.

\section{Advanced Instrumentation}

High-resolution imaging by FM-AFM in liquid requires the use of a relatively stiff cantilever, small cantilever oscillation amplitude and low noise cantilever deflection sensor. In addition, stable operation of FM-AFM and accurate force measurements by FM-AFM require the use of a direct cantilever excitation method such as the magnetic [41] or photothermal [42] excitation method instead of the standard piezoelectric excitation method [43]. Details of such basic instrumentation of liquidenvironment FM-AFM is well summarized in the previous volume of this NC-AFM book series [44]. In this section, we focus on the advanced instrumentation works that we performed since 2007.

\subsection{D Scanning Force Microscopy}

There have been proposed several different techniques for 3D force measurements by FM-AFM. In 2002, Hölscher et al. presented a full 3D force distribution image of a $\mathrm{NiO}$ /vacuum interface obtained in ultrahigh vacuum (UHV) at low temperature 


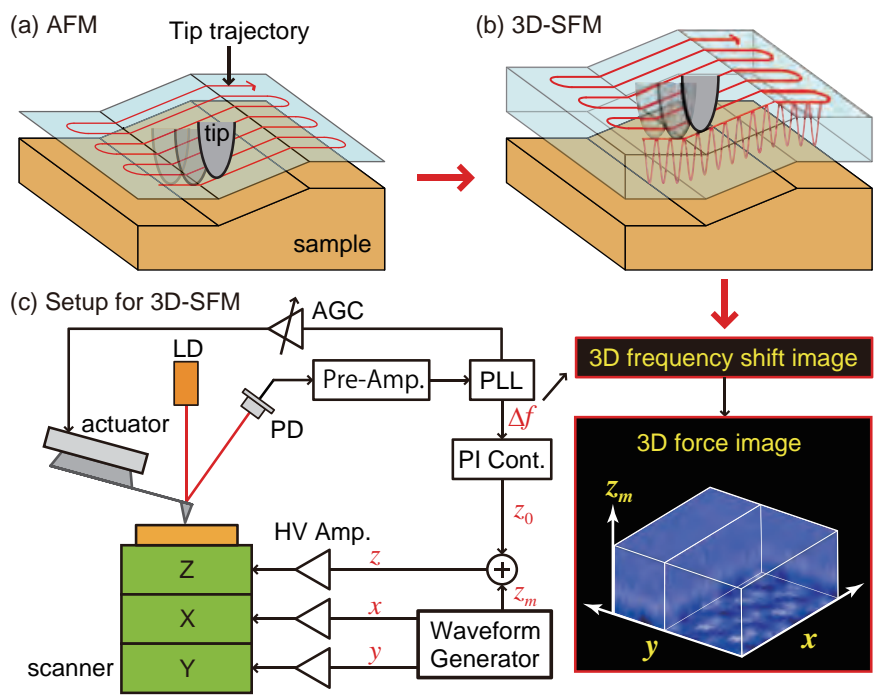

Fig. 1 Basic principles of (a) conventional AFM and (b) 3D-SFM. (c) Experimental setup for the developed 3D-SFM. The inset shows a 3D force image obtained at a mica-water interface $(2 \times 2 \times$ $0.78 \mathrm{~nm}^{3}$ ). (Reused with permission from Fukuma et al [23]. Copyright 2010, American Physical Society.)

[45]. In their method, $\Delta f$ versus distance curves were measured at $32 \times 32$ grid positions with a measurement time of $80 \mathrm{~min}$. In 2009, Albers et al. introduced another method for 3D imaging of a HOPG/vacuum interface obtained in UHV at low temperature [46]. They collected 2D $\Delta f$ images with a constant height mode at different $Z$ positions. Based on the correlation between the obtained $2 \mathrm{D}$ images, they can correct the XY drift of the tip position. Although it took them $40 \mathrm{~h}$ to complete a single 3D imaging, the obtained image has a high pixel density (256 $\times 119$ pixels). In these previous techniques, the imaging time is typically longer than $1 \mathrm{~h}$. This is acceptable for experiments in UHV at low temperature, where thermal drift of the tip position is very small. However, this imaging time is too long for liquid-environment experiments at room temperature, where non-linear drift of the tip position is caused by the swelling of the materials adjacent to the imaging solution.

To overcome this problem, we have developed another 3D force measurement technique referred to as 3D-SFM (Fig. 1) [23]. In conventional AFM, a tip is laterally scanned with the tip-sample distance kept almost constant by the constant $\Delta f$ feedback control (Fig. 1(a)). In 3D-SFM, while a tip is slowly scanned laterally, it is also vertically scanned with a much faster scan speed (Fig. 1(b)). In this way, the tip is scanned in the whole 3D interfacial space. During the tip scan, the fast variation of $\Delta f$ is recorded in real time to produce a 3D $\Delta f$ image. In the meantime, the averaged tip-sample distance is controlled such that the averaged $\Delta f$ is kept constant. In this way, we can avoid a tip crash into the surface even if there is any surface tilt or a tip drift. 
A typical experimental setup for 3D-SFM is schematically shown in Fig. 1(c). The setup is very similar to the one for a standard FM-AFM instrument. Thus, here we explain only the difference from it. For the $Z$ tip scan, we produce a sine wave $\left(z_{m}\right)$ with a modulation frequency much faster than the bandwidth of the tip-sample distance regulation. This signal is added to the output from the feedback controller $\left(z_{0}\right)$. The $\Delta f$ signal from the phase-locked loop (PLL) circuit is recorded to produce a 3D $\Delta f$ image. The obtained image is converted to a 3D force image by SaderJarvis equation [24]. In the meanwhile, $z_{0}$ signal is also recorded to produce a $2 \mathrm{D}$ height image.

Owing to the continuous $Z$ scan signal, 3D-SFM is much faster than the previously proposed 3D measurement methods $[45,46]$. We typically obtain a full 3D $\Delta f$ image in $53 \mathrm{sec}$ with a scan size of $4 \times 4 \times 2 \mathrm{~nm}^{2}$ and a pixel resolution of 64 $\times 64 \times 256$ pixels in $X Y Z$. In spite of the fast scan speed, we can visualize a 3D force distribution with subnanometer-scale resolution (the inset in Fig. 1).

\subsection{Improvements of fundamental performance}

Since 2007, we have been working on the improvement of fundamental performance of FM-AFM such as force sensitivity and operation speed. Recently, we finally obtained some outcome from these efforts, which has significantly expanded the applicability of liquid-environment FM-AFM. Here, I summarize key issues in these developments.

\subsubsection{Force resolution}

True atomic-resolution imaging by FM-AFM in liquid typically requires $F_{\min }$ of $10 \mathrm{pN}$ at $B=100 \mathrm{~Hz}$. Thus, the present FM-AFM barely satisfies this requirement. Such small margin of the performance leads to poor reproducibility or strong dependence on the user skill and often prevents systematic studies by FM-AFM. Furthermore, 3D measurements of hydration structures imposes a more stringent requirement, $F_{\min }$ of $10 \mathrm{pN}$ at $B=1 \mathrm{kHz}$, because of the faster scanning speed in $Z$. This requirement is not fully satisfied by the present FM-AFM and hence it may fail to visualize important features related to the interfacial phenomenon that we are interested in. To solve these problems, we have improved $F_{\min }$ of liquid-environment FM-AFM.

$F_{\min }$ of FM-AFM is approximately given by [1]

$$
F_{\min }=\sqrt{\frac{4 k k_{B} T B}{\pi f_{0} Q}},
$$

where $k_{B}, T, Q$ and $f_{0}$ denote Boltzmann's constant, temperature, $\mathrm{Q}$ factor of the cantilever resonance and cantilever resonance frequency, respectively. Among the 

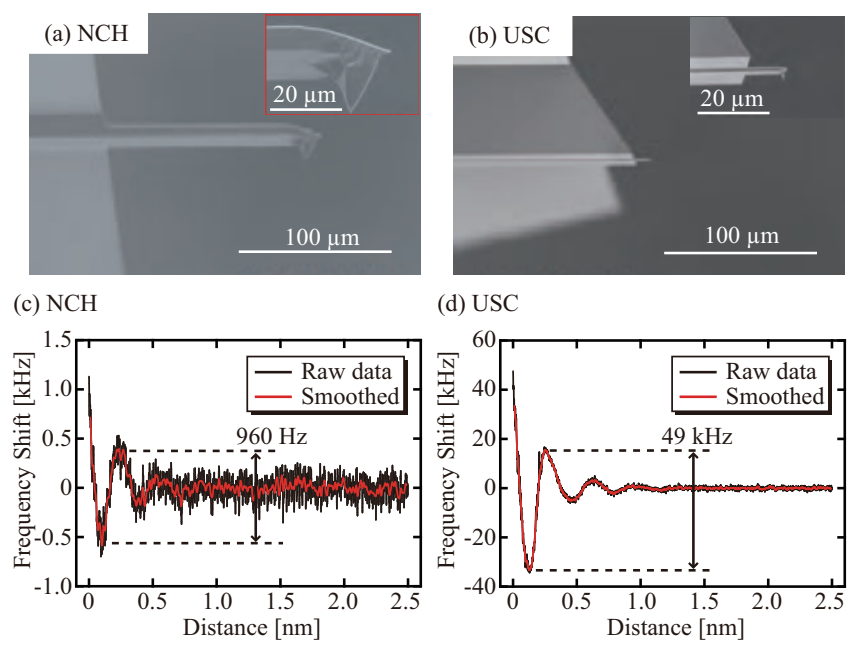

Fig. 2 SEM images of (a) the conventional cantilever (NCH, Nanoworld) and (b) the small cantilever (USC (prototype), Nanoworld). $\Delta f$ versus distance curves measured on mica in PBS solution by (a) NCH and (b) USC cantilevers. (Reused with permission from Fukuma et al [50]. Copyright 2012, Institute of Physics.)

parameters in the right side of this equation, $k_{B}, T$ and $B$ are determined by the requirements for individual applications. Thus, we cannot arbitrarily change it for improving $F_{\min }$. The rest of the parameters are all relevant to the cantilever characteristics. Therefore, $F_{\min }$ is practically determined by the cantilever characteristics.

Among $k, Q$ and $f_{0}$, the only parameter that we can arbitrarily change for improving $F_{\min }$ without deteriorating other performance is $f_{0}$. For increasing $f_{0}$ without causing significant change in $Q$ or $k$, we should reduce the cantilever size in length, width and thickness. This idea is not very new but was proposed in the early stage of the AFM development [47-49]. In spite of the previous efforts, the use of a small cantilever has not been common. This is due to the following technical difficulties. First, it was difficult to produce such small cantilevers with an acceptable cost. Secondly, AFM instruments were not designed for using such a small cantilever.

Recently, this situation has started to change. Major AFM probe manufacturers have established a mass production process of small cantilevers with a reasonable yield rate (e.g. USC, Nanoworld and AC55, Olympus). They recently commercialized these cantilevers with an affordable price. In the meanwhile, we have been working with these companies as a test user and have developed an FM-AFM instrument compatible with the small cantilevers [50].

Figures 2(a) and 2(b) show scanning electron microscopy (SEM) images of the conventional (NCH) and small cantilevers (USC (prototype)) provided by Nanoworld. The SEM images show that the size of the USC cantilever is much smaller than that of $\mathrm{NCH}$. Owing to this small size, $f_{0}$ of USC is as high as $3-4 \mathrm{MHz}$ in water. This is much higher than that of $\mathrm{NCH}$ (typically $120-160 \mathrm{kHz}$ in water). In the meanwhile, 
$Q$ and $k$ of USC are almost the same as those of NCH. From Eq. (1), USC should provide $\sim 5$ times smaller $F_{\min }$ than $\mathrm{NCH}$.

This expectation is true only when we can achieve the optimal performance limited by the thermal cantilever vibration. To achieve this performance, we need to satisfy two technical requirements. First, a cantilever deflection sensor should have sufficiently low noise and wide bandwidth. For detecting the cantilever vibration at 3-4 MHz, the deflection sensor should have a bandwidth higher than $10 \mathrm{MHz}$. We satisfied this requirement by optimizing the circuit design of the preamplifier and the differential amplifier in the deflection sensor [51]. To achieve the thermal-noiselimited performance, the deflection noise density caused by the deflection sensor $\left(n_{z s}\right)$ should be sufficiently smaller than that by the cantilever Brownian motion $\left(n_{z B}\right)$.

$n_{z B}$ is given by

$$
n_{z B}=\sqrt{\frac{2 k_{B} T}{\pi f_{0} k Q} \frac{1}{\left[1-\left(f / f_{0}\right)^{2}\right]^{2}+\left[f /\left(f_{0} Q\right)\right]^{2}}} .
$$

The noise component that influences FM-AFM performance is in the frequence range from $f_{0}-B$ to $f_{0}+B$. In this frequency range, $n_{z B}$ is almost constant due to the small $\mathrm{Q}$ factor. From Eq. (2), the $n_{z B}$ value at $f_{0}$ is given by

$$
n_{z B}\left(f_{0}\right)=\sqrt{\frac{2 k_{B} T Q}{\pi f_{0} k}} .
$$

This value is proportional to $1 / \sqrt{f_{0}}$. Thus, the high $f_{0}$ of the small cantilever makes it difficult to achieve the thermal-noise-limited performance. If we define the required value as the half of $n_{z B}\left(f_{0}\right)$, it is typically $40 \mathrm{fm} / \sqrt{\mathrm{Hz}}$ for $\mathrm{NCH}$ while 10 $\mathrm{fm} / \sqrt{\mathrm{Hz}}$ for USC cantilever.

We satisfied this requirement by developing a low noise cantilever deflection sensor using radio frequency (RF) laser power modulation technique [13] and a replaceable objective lens system [52]. Owing to these improvements, the $n_{z s}$ obtained by our deflection sensor is $\sim 5 \mathrm{fm} / \sqrt{\mathrm{Hz}}$, which is much smaller than the required value for achieving the thermal-noise-limited performance with USC.

The second requirement for achieving the thermal-noise-limited performance is a stable cantilever excitation system. We satisfied this requirement by developing a stable photothermal excitation system. A stable operation of dynamic-mode AFM in liquid and accurate measurements by it generally require a direct cantilever excitation method such as the magnetic [41] or photothermal [42] excitation method. For the small cantilever excitation, the photothermal excitation method is more suitable than the magnetic excitation method. This is because the magnetic force applied to the cantilever is reduced by the reduction of the cantilever size and the generation of a sufficiently strong magnetic field becomes difficult with increasing the drive frequency. Thus, we used the photothermal excitation method.

However, we found that stable excitation of a small cantilever requires a higher stability of the excitation laser beam than that of a conventional cantilever [50]. This 
is because even a small spatial fluctuation of the laser beam position or shape can give a significant influence on the excitation characteristics of a small cantilever. We overcame this problem by improving the stability of the photothermal excitation setup. Before the improvement, a laser diode module and lens system was integrated into the AFM head. In the improved setup, we used a fiber pig-tailed laser beam source that is separeted from the AFM head. The temperature of the laser source was kept constant while the current for driving the laser diode was kept constant by an automatic current constant (ACC) circuit. To transfer the laser light to the AFM head, we used a polarization-maintaining optical fiber, which is known to have a higher immunity to the thermal and mechanical disturbance than a standard singlemode optical fiber. With these improvements, we made it possible to achieve the thermal-noise-limited performance with a USC cantilever.

With the developed cantilever deflection sensor and the photothermal excitation system, we measured $\Delta f$ versus distance curves on mica in phosphate buffered saline (PBS) solution by NCH and USC cantilevers (Figs. 2(c) and 2(d)) [50]. Both the curves show an oscillatory profile corresponding to the hydration structure formed at the mica-water interfaces. This figure shows that USC provides a better signal-to-noise ratio in the force curve measurements. This is due to the significant improvement of the force sensitivity provided by the high $f_{0}$. The $\Delta f$ difference between the minimum and the maximum peak is $960 \mathrm{~Hz}$ for $\mathrm{NCH}$ and 49 $\mathrm{kHz}$ for USC. The result shows that the small cantilever provides $\sim 50$ times improvement in the force sensitivity. However, the enhancement of $f_{0}$ also increases $\Delta f$ noise. Consequently, $F_{\min }$ is improved from $10.7 \mathrm{pN}$ to $1.44 \mathrm{pN}$ for $B=100$ Hz. Therefore, we can achieve approximately seven-fold improvement by the small cantilever. This improvement should give enough margin of the performance in the 2D FM-AFM imaging. In addition, now we have sufficient $F_{\min }$ for $3 \mathrm{D}$ force measurements. Thus, we may be able to visualize important features of 3D hydration structures that have not been accessible by conventional FM-AFM.

\subsubsection{Operation speed}

FM-AFM has been traditionally used for atomic- or molecular-resolution imaging of various materials in vacuum [3]. For those applications, it has not been very important to have a high operation speed. However, at solid-liquid interfaces, surface structures are often dynamically changing. In addition, there have been growing interests in dynamic processes such as biomolecular interactions and electrochemical reactions. To visualize these dynamic processes, we should increase the operation speed of FM-AFM. Even for the imaging of static surface structures, biological applications often require a wider feedback bandwidth of the tip-sample distance regulation. This is because the surface corrugations and inhomogeneities of biological samples are much larger than those of atomically or molecularly flat samples that have been investigated by FM-AFM in vacuum. To solve these problems, we have improved the operation speed of FM-AFM. 


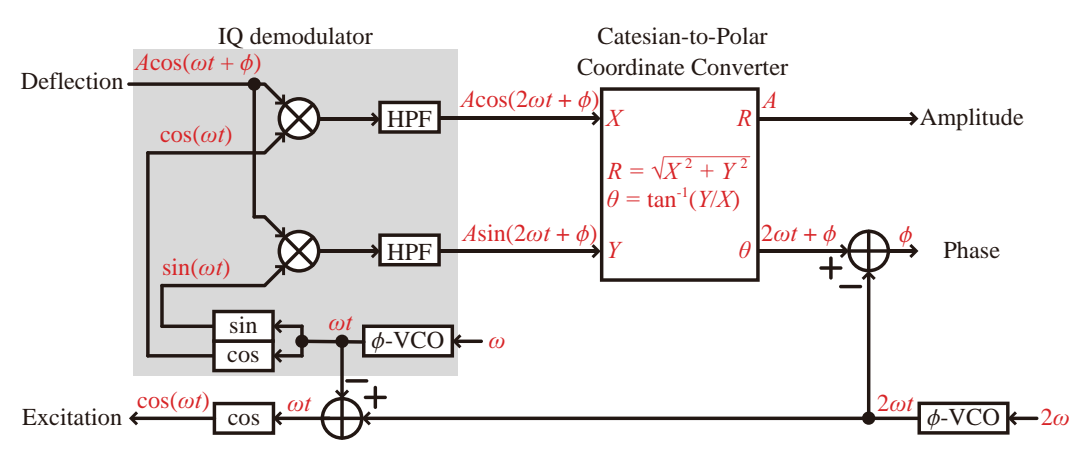

Fig. 3 Schematic diagram of the developed PD. (Reused with permission from Miyata et al [55]. Copyright 2013, American Institute of Physics.)

To increase the measurement bandwidth $B$ of FM-AFM without deteriorating other performance, we need to satisfy the following two requirements. Firstly, we should have sufficient $F_{\min }$ for a given $B$. We satisfied this requirement by using the small cantilever [50]. As discussed above, the small cantilever gives seven-fold improvement in $F_{\min }$ for a given $B$. From Eq. (1), this implies that $B$ can be increased by 50 times for a given $F_{\min }$. We can operate a conventional FM-AFM with $F_{\min }=$ $10 \mathrm{pN}$ at $B=100 \mathrm{~Hz}$ and obtain an atomic-resolution image at $1 \mathrm{~min} / \mathrm{frame}$. Thus, with the small cantilever, we should be able to operate FM-AFM with $F_{\min }=10 \mathrm{pN}$ at $B=5 \mathrm{kHz}$ and obtain an atomic-resolution image at $1 \mathrm{sec} / \mathrm{frame}$.

Secondly, to perform such a fast imaging, we need to increase the bandwidth and to reduce the latency of all the components constituting an FM-AFM. To satisfy this requirement, we improved most of the components in our FM-AFM setup. We improved the circuit design of the cantilever deflection sensor and improved its bandwidth to $\sim 10 \mathrm{MHz}$ [51]. We replaced the piezoelectric excitation mechanism with the photothermal excitation system and achieved over $10 \mathrm{MHz}$ bandwidth [51]. We developed a separate-type high-speed $X Y$ sample and $Z$ tip scanner and enabled their operation at $\sim 1 \mathrm{kHz}$ and $\sim 10 \mathrm{kHz}$, respectively [53]. We also developed a high-speed and low noise high-voltage amplifier that can achieve 200 $\mathrm{kHz}$ bandwidth with a $100 \mathrm{nF}$ capacitive load [53], which is a typical capacitance of a piezoelectric actuator used in an AFM scanner. We also improved the bandwidth and the latency of the PLL circuit used for $\Delta f$ detection and cantilever oscillation control $[54,55]$.

Among these components, the PLL circuit is the core part of an FM-AFM setup. In the improvement of the PLL circuit, we proposed a unique digital signal processing algorithm for implementing a high-speed phase detector (PD) [55]. Figure 3 shows a schematic diagram of the developed PD. In this design, the cantilever deflection signal $A \cos (\omega t+\phi)$ is fed into the IQ demodulator. $\omega$ and $\phi$ denote frequency and phase of the cantilever oscillation, respectively. In the IQ demodulator, the input signal is multiplied by the reference signals $[\cos (\omega t)$ and $\sin (\omega t)]$ and converted to dc and $2 \omega$ components. While the dc components are eliminated by the high-pass filters (HPFs), the $2 \omega$ components $[A \cos (2 \omega t+\phi)$ and $A \sin (2 \omega t+\phi)]$ 

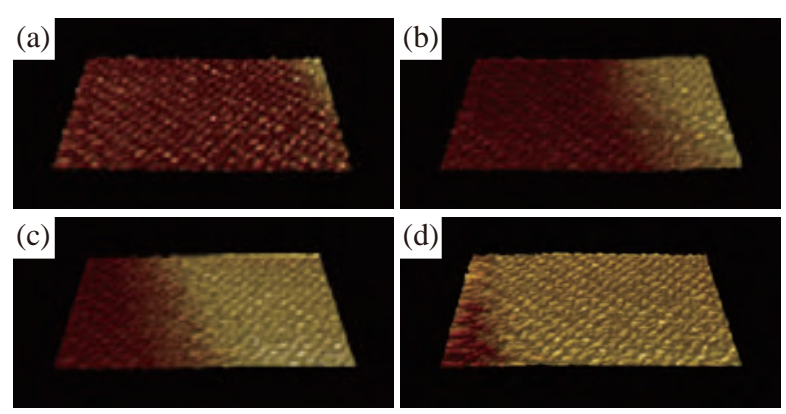

Fig. 4 Phase modulation AFM images of the cleaved calcite surface obtained in water. The images were selected from the 44 successive images obtained at $1 \mathrm{~s} /$ frame. (a) $0 \mathrm{~s}$. (b) $5 \mathrm{~s}$. (c) $10 \mathrm{~s}$. (d) 15 s. Scan size: $10 \times 5 \mathrm{~nm}^{2}$. Scan rate: $100 \mathrm{~Hz}$. Pixel size: $200 \times 100 \mathrm{pix}^{2}$. (Reused with permission from Miyata et al. [55] Copyright 2013, American Institute of Physics.)

are passed to a Cartesian-to-Polar coordinate converter. In the circuit, amplitude $(A)$ and phase $(2 \omega t+\phi)$ of the complex signal are calculated.

In the developed PD, phase signals, $\omega t$ and $2 \omega t$, are generated by phase-output voltage controlled oscillators ( $\phi$-VCOs). The $\omega t$ signal is fed into sine and cosine converters to produce the two reference signals. The $2 \omega t$ signal is subtracted from the $(2 \omega t+\phi)$ signal to produce the final output of the PD $(\phi)$. The difference between the $2 \omega t$ and $\omega t$ signals is calculated and fed into a cosine converter to produce the cantilever excitation signal $[\cos (\omega t)]$.

We implemented the proposed circuit in a field programmable gate array (FPGA) chip (Vertex-5, Xilinx). The clock frequency of the FPGA was set at $100 \mathrm{MHz}$. The sampling rates of the analog-to-digital and digital-to-analog converters were set at 100 MSPS.

In the proposed algorithm, the significant improvement is achieved by the HPFs. If we use a small cantilever with a $f_{0}$ of $3 \mathrm{MHz}$, the deflection signal is converted to the dc and $6 \mathrm{MHz}$ components. While the dc components are eliminated by the HPFs, $6 \mathrm{MHz}$ components are passed through the HPFs. At the pass band frequency, the HPFs have only 0.13 clk group delay. Therefore, this algorithm enables IQ demodulation with almost no delay. This is a remarkable improvement from the conventional PD, which utilizes a multiplier and low-pass filters (LPFs).

The developed PD has a bandwidth of $3 \mathrm{MHz}$, which is the same frequency as the input signal. Namely, the bandwidth of the PD is determined by the input signal frequency at least for a signal slower than $10 \mathrm{MHz}$. However, this does not mean that the circuit has an infinite speed. In fact, the circuit causes a finite delay, which is referred to as latency. The latency of the PD for a $3 \mathrm{MHz}$ input signal is $0.97 \mu \mathrm{s}$. This latency causes only $1.8^{\circ}$ phase delay at $5 \mathrm{kHz}$. Thus, the PD is fast enough to achieve $5 \mathrm{kHz} B$ in the AFM measurement.

Figure 4 shows high-speed phase modulation AFM (PM-AFM) images of a cleaved calcite surface obtained in water using the developed PD. These images are selected from the 44 successive images taken at $1 \mathrm{sec} / \mathrm{frame}$. In the images, the lateral motion of the step edge caused by the crystal growth is clearly visualized with 
atomic resolution. The distance between the adjacent atomic-scale corrugations is $\sim 0.5 \mathrm{~nm}$ while the tip velocity is $2 \mu \mathrm{m} / \mathrm{s}$. Thus, the frequency of the corrugations to be detected $\left(f_{\text {cr }}\right)$ is $\sim 4 \mathrm{kHz}$. This is close to the maximum $B(5 \mathrm{kHz})$ that we can achieve without deteriorating $F_{\min }$.

In the above application example, we used the developed PD for operating PMAFM. We also developed a PLL circuit using the high-speed PD and obtained some preliminary results. Therefore, the proposed PD is also applicable to the high-speed operation of FM-AFM. The developed high-speed AFM should enable in-situ imaging of various interfacial phenomena such as crystal growth, dissolution and corrosion processes with atomic-scale resolution.

\section{Applications of liquid-environment FM-AFM}

Since 2005, FM-AFM has been used for subnanometer-scale imaging of various surfaces. Some of the early works are summarized in the previous volume of this NC-AFM book series $[3,44]$. In this chapter, I focus on the recent applications that we reported after the publication of the previous volume in 2009.

\section{$3.12 D$ imaging}

After the first demonstration of the true atomic-resolution imaging by FM-AFM in liquid, the method has been used for the imaging of various materials including minerals, biological systems and other organic molecules. Among these applications, here we present the imaging studies of lipid-cholesterol complexes [22], tubulin protofilaments [56] and $\mathrm{CaF}_{2}(111)$ surfaces [25].

\subsubsection{Lipid-cholesterol mixed bilayers}

Cholesterol is one of the major components of biological membranes. It is known to influence various membrane properties such as elasticity, mechanical strength and molecular fluidity [57,58]. Local variations in the membrane properties caused by the existence or absence of cholesterols are closely related to the formation of nanoscale domains referred to as lipid rafts. The existence of lipid rafts with different affinity to membrane proteins may provide a driving force for protein trafficking or present a local environment that is favorable to specific protein function [59].

To date, biological and physical roles of cholesterols have been intensively studied using a lipid bilayer as a model biological membrane. However, it has been difficult to clarify molecular-scale behavior of the cholesterols and discuss its influence on global membrane properties. For example, studies by nuclear magnetic resonance (NMR) [60] suggested that the cholesterol molecules are inserted between 


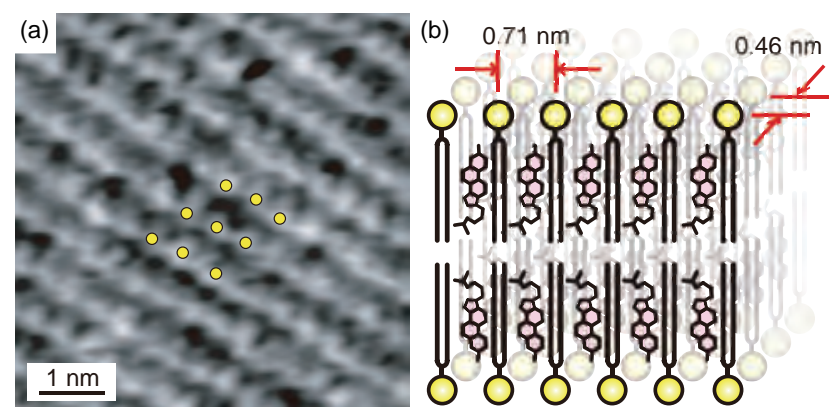

Fig. 5 (a) FM-AFM image of a DPPC-cholesterol (1:1) mixed bilayer obtained in PBS solution. Scan size: $5 \mathrm{~nm} \times 5 \mathrm{~nm}$. $A=0.19 \mathrm{~nm} . \Delta f=+207 \mathrm{~Hz}$. Tip velocity: $200 \mathrm{~nm} / \mathrm{s} . k=33.4 \mathrm{~N} / \mathrm{m}$. Image height: $60 \mathrm{pm}$. (b) Proposed model for the DPPC-cholesterol (1:1) mixed bilayer. (Reused with permission from Asakawa et al [22]. Copyright 2009, Institute of Physics.)

lipid molecules to induce conformational changes in lipid acyl chains. However, the real-space description of local structure formed by the cholesterol-lipid interaction has not been understood due to the lack of a method able to directly visualize the molecular-scale structures in real space.

We investigated mixed dipalmitoylphosphatidylcholine (DPPC) and cholesterol (1:1) bilayers by FM-AFM. Figure 5(a) shows an FM-AFM image of the DPPCcholesterol bilayer. The image shows the stripe features separated by $0.71 \mathrm{~nm}$. Individual stripes consists of DPPC molecules separated by $0.46 \mathrm{~nm}$. The image reveals that the mixed bilayer has a rhombic molecular arrangement. This is a clear difference from the molecular arrangement in a DPPC bilayer, where molecules are hexagonally packed with a spacing of $\sim 0.5 \mathrm{~nm}$.

A previous study using MD simulation [61] reported that the lateral spacing of DPPC molecules is expanded by inserting cholesterols between the acyl chains of DPPC molecules. An important finding of our experiment is that a DPPCcholesterol bilayer exhibits an anisotropic packing, whereas a pure DPPC bilayer presents a hexagonal pseudo-isotropic packing. The result indicated that the inserted cholesterol molecules are linearly aligned between the DPPC molecules in the direction as shown in Fig. 5(b). So far, such a linear arrangement of the inserted cholesterols has been tentatively suggested by some of the simulation studies as a possible short-range ordering [62]. Although 50\% molar content of cholesterol is much larger than that in the physiological conditions, our result clarified that the DPPC-cholesterol mixture favors such an arrangement when sufficient number of cholesterols exist in the local area of lipid bilayer.

\subsubsection{Tubulin protofilaments}

Tubulins are globular proteins with a diameter of approximately $4 \mathrm{~nm}$ [63]. The $\alpha \beta$-tubulin heterodimers are known as the smallest unit of building blocks of microtubules, which are one of the components of the cytoskeleton with a diameter of 

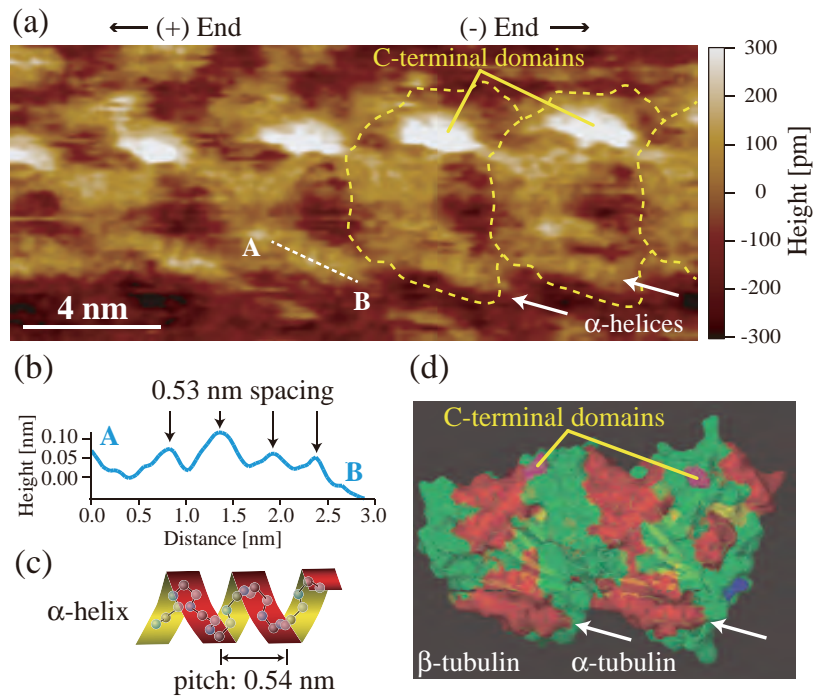

(d)

Fig. 6 (a) FM-AFM image of a tubulin protofilament in a sheet-like structure $(\Delta f=+3.0 \mathrm{~Hz}$, $A=0.30 \mathrm{~nm}$, tip velocity: $100 \mathrm{~nm} / \mathrm{s}$ ). (b) Height profile measured along line A-B in panel a (average spacing: $0.53 \mathrm{~nm}$, standard deviation: $0.056, n=12$ ). (c) Schematic illustration of a-helix backbone. (d) Structural model of a tubulin heterodimer (PDB ID: 1JFF). (Reused with permission from Asakawa et al [56]. Copyright 2011, Biophysical Society.)

$25 \mathrm{~nm}$. Microtubules serve as structural components within cells and are involved in many cellular processes. For example, microtubules act as a molecular rail for motor proteins, such as kinesin and dynein, in intracellular transportation. Previous studies have demonstrated that the carboxyl-terminal (C-terminal) domain of tubulin has strong influence on the interaction with these motor proteins. The interactions between tubulins and motor proteins are regulated by post-translational modifications of the C-terminal tails [64]. In spite of the importance, detailed structure of tubulin $\mathrm{C}$-termini and its relation to their functions have not been clarified even with X-ray or NMR studies [63].

We have investigated the tubulin assemblies by FM-AFM in liquid to visualize local secondary structures, including the C-terminal tails [56]. In Fig. 6(a), one of the protofilaments constituting a sheet-like structure was imaged. The image shows nanoscale repeating structures corresponding to the tubulin monomers as well as subnanometer-scale features within each molecule. In particular, the fine features indicated by the arrows in Fig. 6(a) exhibit a periodic corrugation with a spacing of $0.53 \mathrm{~nm}$, as revealed by a cross-sectional plot taken along line A-B (Fig. 6(b)). This average spacing is in agreement with the common pitch of an $\alpha$-helix backbone $(0.54 \mathrm{~nm})$, as illustrated in Fig. 6(c), which strongly suggests that the periodic features correspond to $\alpha$-helices at the tubulin surface.

The direct imaging of $\alpha$-helices provides useful information for identifying the orientation of a tubulin protofilament. Although the structural model of a tubulin 
protofilament is known, to interpret the subnanometer-scale features in an FM-AFM image, one must determine the rotation angle of the protofilament. We compared the subnanometer-resolution FM-AFM image (Fig. 6(a)) with the known structural model of the tubulin heterodimer (PDB ID: 1JFF) with various rotation angles. As a result, we found that the arrangement of the observed $\alpha$-helices exhibits the best agreement with that of the structural model when the rotation angle is set as shown in Fig. 6(d). The red regions of the structural model represent $\alpha$-helices at the surface of tubulins. Although it is difficult to distinguish between $\alpha$-and $\beta$-tubulins in the FM-AFM image, the result allowed us to determine the directions of the $(+)$ and (-) ends of the tubulin protofilament, as shown in Fig. 6(a). Moreover, the good agreement between the AFM image and the structural model allowed us to interpret local features in the high-resolution FM-AFM image. The positions of the bright protrusions observed in the FM-AFM image (Fig. 6(a)) agree with those of the Cterminal domains in the structural model (Fig. 6(d)). This result demonstrates that the C-terminal domains of tubulins can be directly imaged by FM-AFM in liquid.

The results obtained in this study demonstrate that FM-AFM is capable of identifying arrangements of secondary structures such as $\alpha$-helices and C-terminal domains at the tubulin surface. This capability should be important in future studies on the molecular-scale mechanisms of tubulin functions.

\subsection{3 $\mathrm{CaF}_{2}(111)$ surface}

Fluorite $\left(\mathrm{CaF}_{2}\right)$ crystals are widely used for various industrial applications. Examples include optical components for semiconductor lithography and other laser technologies $[65,66]$ and scintillator for radioactivity investigations $[67,68]$. Thus, the crystal growth and dissolution processes of fluorite have intensively been studied to improve the crystal quality $[69,70]$. In addition, these processes play important roles in the formation of tooth enamel and pathogenesis of dental caries [71,72], as well as in the desalination for oil and gas recovery [73-75] and water purification [76-78]. Therefore, the physicochemical processes taking place at fluorite-water interface are important in industrial, environmental and medical research fields.

AFM has widely been used for investigating crystal growth and dissolution processes of various minerals. So far, Hillner et al. imaged crystal growth of a fluorite(111) surface in supersaturated solution by contact-mode AFM [79]. They reported the vertical growth of sharp asperities with a height of $10-500 \mathrm{~nm}$ on a flat terrace. Subsequently, Bosbach et al. imaged a fluorite(111) surface in an undersaturated solution by contact-mode AFM [80]. They found that the dissolution takes place by the formation of pits on terraces followed by the retreat of step edges. They also found the formation of nanoscale protrusions. Jordan et al. investigated the formation mechanism and the composition of the nanoscale protrusions by imaging fluorite(111) surface in various solutions with different $\mathrm{pH}$ [81]. Based on the results, they suggested that the protrusions correspond to multinuclear calcium (aquo) hydroxo complexes $\left[\mathrm{Ca}_{x}(\mathrm{OH})_{y}^{2 x-y}(\mathrm{aq})\right]$ connected to surface hydroxyl groups. 
The previous research clarified nanoscale behavior of fluorite(111) surface in water such as the crystal growth and dissolution, and the formation of the protrusions. However, to understand the mechanism of these processes, we should also clarify atomic-scale interfacial phenomena such as adsorption or weak interaction of ions or other chemical species in the interfacial space with the crystal surface. Due to the limited resolution and the relatively large loading force of the conventional AFM, it has been difficult to visualize such delicate atomic-scale interfacial phenomena.

Table 1 Summary of the interfacial phenomena observed in various aqueous solutions.

\begin{tabular}{lcccc}
\hline & \multicolumn{2}{c}{ Crystal Growth Protrusions } & Adsorbates & Protons \\
\hline Water (pH: 6.5) & Dissolution & Yes & Yes & No \\
Acidified water (pH: 2) & Dissolution & No & No & Yes \\
Saturated (pH: 6) & Equilibrium & Yes & Yes & No \\
Acidified Saturated $(\mathrm{pH}: 2)$ & Equilibrium & No & No & Yes \\
Supersaturated $(\mathrm{pH}: 6, \sigma: 10)$ & Growth & No & Yes & No \\
Supersaturated $(\mathrm{pH}: 6, \sigma: 100)$ & Growth & No & No & No \\
\hline
\end{tabular}

We have investigated atomic-scale processes taking place at fluorite-water interface [25]. We performed FM-AFM imaging of the fluorite(111) surface in various solutions including water $(\mathrm{pH}=2$ and 6.5), saturated solution $(\mathrm{pH}=2$ and 6$)$ and supersaturated solution ( $\mathrm{pH}=6, \sigma=10$ and 100). The observed interfacial phenomena are summarized in Table 1. By analyzing the results, we obtained the following three major findings.

First, we found that the atomic-scale surface roughening takes place at low $\mathrm{pH}$ probably due to the proton adsorption as shown in Figure 7(a). In the previous report, the increase of H-bonds on fluorite(111) surface has been suggested based on the results obtained by the force curve measurements [81]. The atomic-scale images obtained in this study further strengthened this idea and provided a real-space description of this interfacial phenomenon.

Secondly, we found the existence of the surface adsorbates at high $\mathrm{pH}$ as indicated by the arrow A in Fig. 7(b). Based on their behavior, we consider that they are most likely to be calcium hydroxo complexes. Unlike the nanoscale protrusions indicated by the arrow B in Fig. 7(b), the lateral distribution of the surface adsorbates is not limited to the vicinity of the $\mathrm{F}^{-}$vacancies. This is because they are not connected to the surface hydroxyl groups but weakly interact with the fluorite surface.

Finally, we found that the increase of fluorite concentration in solution can prevent the formation of the calcium hydroxo complexes, as shown in Figs. 7(c) and 7(d). The increase of the fluorite concentration results in the increase of $\mathrm{Ca}^{2+}$ and $\mathrm{F}^{-}$ and the decrease of $\mathrm{OH}^{-}$concentrations in the electric double layer. Consequently, the formation of the calcium hydroxo complexes is suppressed. In addition, we suggest that the increased concentration of $\mathrm{F}^{-}$ions in the electric double layer fills 

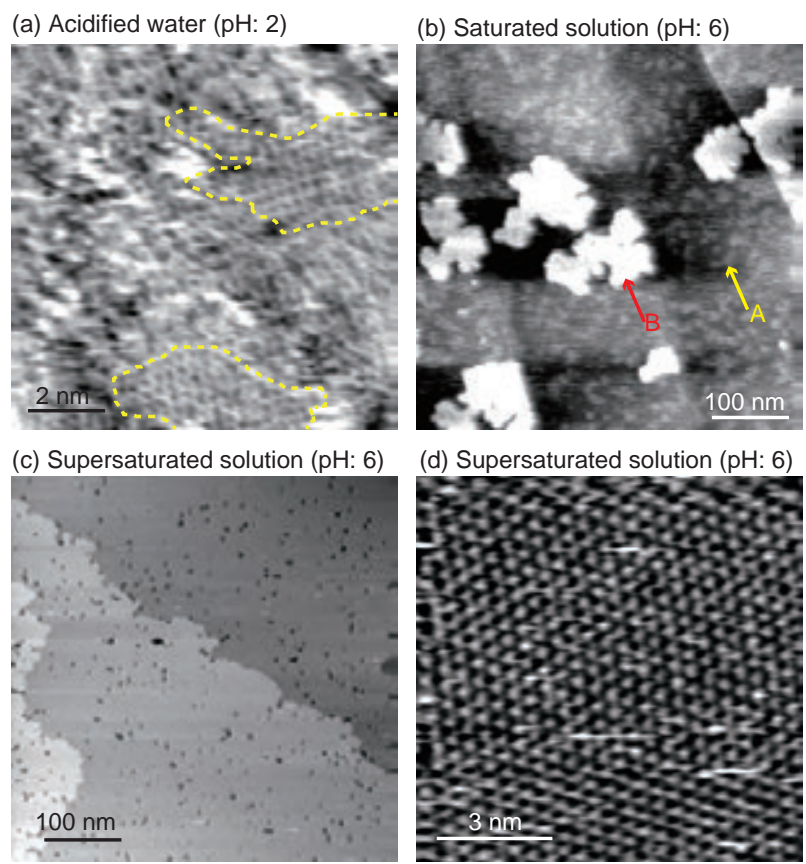

Fig. 7 FM-AFM images of fluorite(111) surfaces in various solution. (a) In acidified water (pH: 2). (b) In saturated solution (pH: 6). (c,d) In supersaturated solution (pH: 6). (Reused with permission from Kobayashi et al [25]. Copyright 2013, American Chemical Society.)

in the surface $\mathrm{F}^{-}$vacancies, which prevents the formation of the surface hydroxyl groups and hence the nanoscale protrusions.

These findings improve our understanding of the interfacial phenomena taking place at the fluorite-water interface. So far, such an atomic-scale study of the interfacial phenomena has been hindered by the limited performance of the conventional AFM. Owing to the high spatial resolution and the small loading force of FM-AFM, we were able to directly visualize atomic-scale interfacial phenomena. This capability should also be useful in other studies on various interfacial phenomena such as crystal growth and dissolution, catalytic reactions and corrosion processes. Therefore, the results obtained here should stimulate the future applications of FM-AFM to the studies in physical chemistry.

\subsection{D Imaging}

In 2010, we demonstrated the first 3D force distribution imaging at mica-water interface [23]. The result shows the possibility of direct imaging of 3D hydration structures with subnanometer-scale resolution. In addition, we applied this technique to 

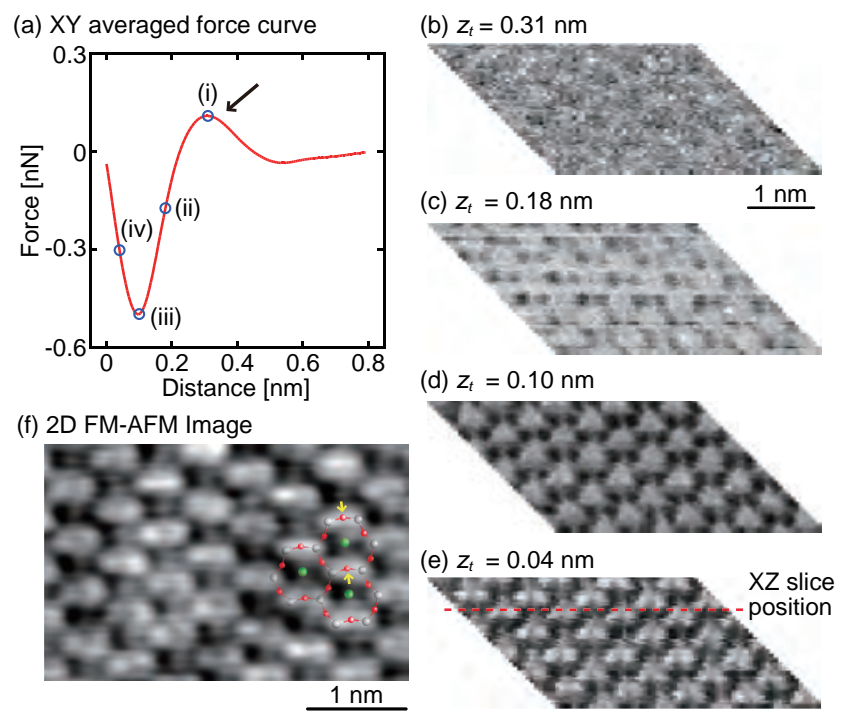

Fig. 8 3D- and 2D images of mica-water interface obtained in PBS solution. (a) $X Y$ averaged force curve. The position for the zero $Z$ tip position $\left(z_{t}=0\right)$ is arbitrary. (b)-(e) $X Y$ cross sections of the 3D-SFM image at $z_{t}=0.31,0.18,0.10$ and $0.04 \mathrm{~nm}$, which respectively correspond to the $z_{t}$ positions indicated by Circles (i)-(iv) in (a). A linear drift correction was applied to the $X Y$ cross sections so that the periodic contrasts match the known lattice constants of a cleaved mica surface. The dotted line in (e) indicates the $Y$ position of the $X Z$ cross section shown in Fig. 9(a). (f) $2 \mathrm{D}$ FM-AFM image $(A=0.26 \mathrm{~nm}, \Delta f=67.1 \mathrm{~Hz})$ obtained with a different tip from the one used for the 3D-SFM imaging. (Reused with permission from Fukuma et al [23]. Copyright 2010, American Physical Society.)

investigate a lipid-water interface that has a much softer interfacial structures [26]. The obtained result suggested the possibility of 3D imaging flexible surface structures such as lipid headgroups. Here, I summarize main issues in the two applications.

\subsubsection{Mica-water interface}

Muscovite mica is known as a prototype of clay minerals and hence has importance in fundamental research regarding clay swelling in geological science [82-84] and cloud seeding in ecological science $[85,86]$. In addition, owing to the ease of cleavage to present an atomically flat surface, mica-water interface has been widely used as a model system to investigate nanofluidics in engineering and physics [87], lubrication in tribology, and molecular adsorption and diffusion in biology and chemistry. To date, water distribution at mica-water interface has been extensively studied by various techniques [88-94]. However, its atomistic model has not been established due to the difficulties in visualizing molecular-scale water distribution directly at a solid-liquid interface. 
We have investigated the hydration structures formed at a mica-water interface by 3D-SFM [23]. From the measured 3D force distribution, an $X Y$ averaged force curve was obtained by plotting force values averaged over an $X Y$ cross section at each $Z$ tip position $z_{t}$ [Fig. 8(a)]. This curve shows an oscillatory profile with a peak [arrow in Fig. 8(a)] having a width of $0.2-0.3 \mathrm{~nm}$, which agrees with the diameter of a water molecule. Owing to this agreement and previous studies on mica-water interface $[12,95]$, we attributed the repulsive peak to the interaction with a hydration layer.

From $X Y$ cross sections at different $z_{t}$ [Fig. 8(b)-(e)], continuous $z_{t}$ dependence of $X Y$ force distribution is obtained. The $X Y$ cross section in Fig. 8(b) does not show any contrast, which reveals the uniform lateral distribution of water in the hydration layer. As the tip approaches the surface, the $X Y$ cross section shows an atomic-scale contrast [Figs. 8(c)-(e)], reflecting the appearance of the short-range interaction force between the tip front atom and the atoms constituting the mica surface. With further decrease of $z_{t}$, the hexagonally arranged force peaks found in Fig. 8(d) change to the pairs of smaller peaks [Fig. 8(e)].

The periodic pairs of the force peaks found in Fig. 8(e) appear to be uniform, which gives rise to a question whether the contrast represents the structure of mica surface or the tip apex [96]. However, we confirmed that a similar contrast is reproduced in a 2D FM-AFM image obtained with a different tip as shown in Fig. 8(f). In the image, the height of individual atoms has irregular variations, which strongly suggests that the contrast is unlikely to be originated from a tip artifact. By comparing the image shown in Fig. 8(f) and the atomic-scale model of mica, we attributed the observed pairs of force peaks to the repulsive forces measured on the two adjacent $\mathrm{Si}$ atoms as indicated by the model overlaid on the image in Fig. 8(f). The height variations of the individual peaks are likely to represent the difference between $\mathrm{Si}$ and $\mathrm{Al}$ atoms as reported previously [12].

A remarkable feature found in the images is an enhanced contrast at the center of the cavity surrounded by a hexagonal ring. The overlaid model shown in Fig. 8(f) reveals that the $X Y$ position of the enhanced contrast corresponds to that of a $\mathrm{OH}$ group located at the bottom of the cavity. The vertical corrugation of the mica surface makes it difficult to analyze correlation between the atomic-scale structure and the force distribution with a 2D FM-AFM image having no vertical extent. Such an analysis becomes possible by extracting a vertical cross section from a 3D-SFM image as shown in Fig. 9(a). The left half of the $X Z$ cross section is shown with a low contrast to visualize the localized force distribution above a $\mathrm{OH}$ group while the right half of the image is shown with a high contrast to visualize the layer-like force distribution over the entire surface. The layer-like force distribution corresponds to the repulsive peak indicated by the arrow in Fig. 8(a) and has been attributed to the hydration layer.

Extracting individual $Z$ profiles constituting the $X Z$ cross section, we obtained site specific force profiles as shown in Fig. 9(b). The $Z$ profiles show strong site dependence especially at the $Z$ distance range $z_{t}<0.2 \mathrm{~nm}$. Profile 1 shows a large attractive force due to the absence of an underlying atom. Profile 2 shows a relatively broad repulsive peak due to the localized force distribution above an $\mathrm{OH}$ group. 
(a) $X Z$ cross section

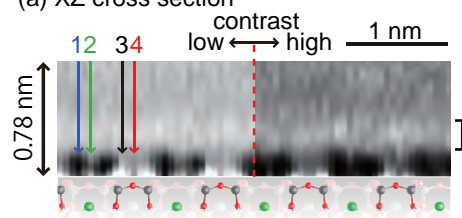

(b) Z profiles

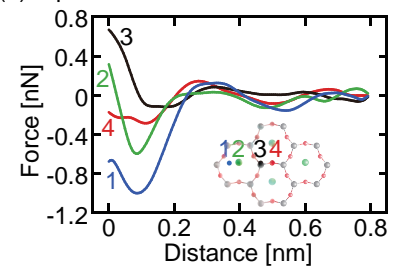

(d) Peak fitting

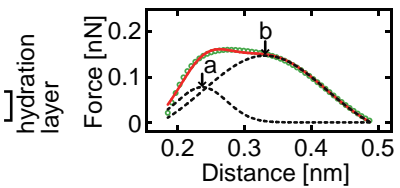

(c) Averaged Z profile

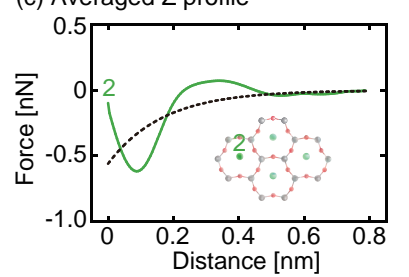

Fig. $9 X Z$ cross section and $Z$ profiles of 3D-SFM image of mica-water interface obtained in PBS solution. (a) $X Z$ cross section obtained at the $Y$ position indicated by a dotted line in Fig. 8(e). An atomic-scale model of [110] projection of muscovite mica is shown below the $X Z$ cross section. (b) $Z$ profiles measured along Line 1-4 indicated in (a). (c) An average of $48 Z$ profiles measured on $\mathrm{OH}$ sites. The dotted line was obtained by fitting the curve with an exponential function. The insets in (b) and (c) show the measurement positions for the $Z$ profiles. (d) The circles show the peak profile obtained by subtracting the dotted line in (c) from the solid line in (c). The dotted lines show the double Gaussian peak profiles obtained by fitting the peak profile. $z_{t}$ values for Peaks $a$ and $b$ are 0.237 and $0.331 \mathrm{~nm}$, respectively. The solid line shows summation of the two dotted lines. (Reused with permission from Fukuma et al [23]. Copyright 2010, American Physical Society.)

On the contrary, Profile 3 shows a shallow and broad attractive peak due to the competition between the attractive van der Waals or hydration force and a repulsive interaction force between the tip and the $\mathrm{Si}$ atom. This means that the repulsive force component measured on the Si site starts to increase at a higher $z_{t}$ than it does on the $\mathrm{OH}$ site owing to their height difference.

Profile 2 shows a relatively broad repulsive peak due to the influence of the localized force distribution above an $\mathrm{OH}$ group. To quantitatively analyze the peak profile, $48 \mathrm{Z}$ profiles measured on $\mathrm{OH}$ sites are taken from the 3D-SFM image and averaged to obtain a smoothed profile [Fig. 9(c)]. The averaged curve was fitted to an exponential function to obtain a long-range background component as indicated by the dotted line in Fig. 9(c). This long-range component is subtracted from the averaged force profile to obtain the peak profile as shown in Fig. 9(d). The peak shows a broad profile with a plateau on top of it, which does not appear to be a single peak but a summation of double peaks. Thus, we fitted the peak profile to a double Gaussian distribution function as indicated by the dotted lines in Fig. 9(d). According to the fitting parameters, the minor peak at $z_{t}=0.237 \mathrm{~nm}$ (Peak $a$ ) has a width of $0.103 \mathrm{~nm}$ while the major peak at $z_{t}=0.331 \mathrm{~nm}$ (Peak $b$ ) has a width of $0.258 \mathrm{~nm}$.

So far, some of the previous studies on the mica-water interface supported the existence of "ice-like" water [88-90] while others suggested the existence of more disordered "liquid-like" water [91,92]. Recently, studies using x-ray reflectome- 
try [93] and Monte Carlo simulation [94] consistently suggested the existence of adsorbed water molecules presenting localized water distribution above $\mathrm{OH}$ groups in addition to the laterally distributed hydration layer. The water density profiles obtained in these previous studies revealed the $Z$ distance between the peaks corresponding to the hydration layer and adsorbed water molecules is $0.12 \mathrm{~nm}$. This value approximately agrees with the peak distance $(0.094 \mathrm{~nm})$ measured in Fig. 9(d), which indicates that the enhanced contrast measured on $\mathrm{OH}$ sites should represent the localized distribution of adsorbed water molecules.

Therefore, the results obtained in this study support the model proposed by Cheng et al. [93], where the adsorbed and laterally-distributed water molecules coexists at the interface. The co-existence of water molecules having a long relaxation time (adsorbed water) and laterally-distributed disordered water molecules (2D hydration layer) may reconcile the two opposing ideas of "ice-like" and "liquid-like" water molecules at the mica-water interface.

\subsubsection{Lipid-water interface}

At the interface between a biological system and its surrounding physiological solution, water molecules interact with biomolecules constituting the surface. Through the interaction, water molecules give significant influence on the structure and function of biomolecules and their assembly [97-99]. Therefore, understanding of the structure and function of a biological system requires investigations on the behavior of interfacial water.

The surface of a biological membrane mainly consists of hydrophilic lipid headgroups. So far, the membrane-water interface has been extensively studied by various techniques $[17,19,100-105]$. These previous works have shown that the water molecules adjacent to a membrane strongly interact with the headgroups and give significant influence on its mechanical strength and fluidity [106-108]. However, molecular-scale origin for such influence has remained elusive.

One of the major difficulties in such a study lies in the measurement of molecularscale structure of a membrane-water interface. As the lipid headgroups exhibit thermal fluctuations, the surface structure of a membrane is inherently ill-defined. In addition, the fluctuating lipid headgroups interact with mobile water, through which the interfacial water presents non-uniform density distribution known as hydration structure. Thus, the understanding of the whole structure of membrane-water interface should require a method to visualize 3D distribution of mobile water as well as fluctuating lipid headgroups.

We have investigated the lipid-water interface by 3D-SFM [26]. We measured a 3D force distribution at the interface between fluctuating lipid headgroups and HEPES buffer solution (Fig. 10(a)). The obtained 3D image showed molecular-scale tilted contrasts reflecting the averaged conformation of the headgroups as well as layer-like contrasts corresponding to the hydration layer (Figs. 10(b) and 10(c)). The results show that we can visualize averaged 3D distribution of fluctuating surface structures as well as that of mobile water (i.e. hydration structure). 
(a)
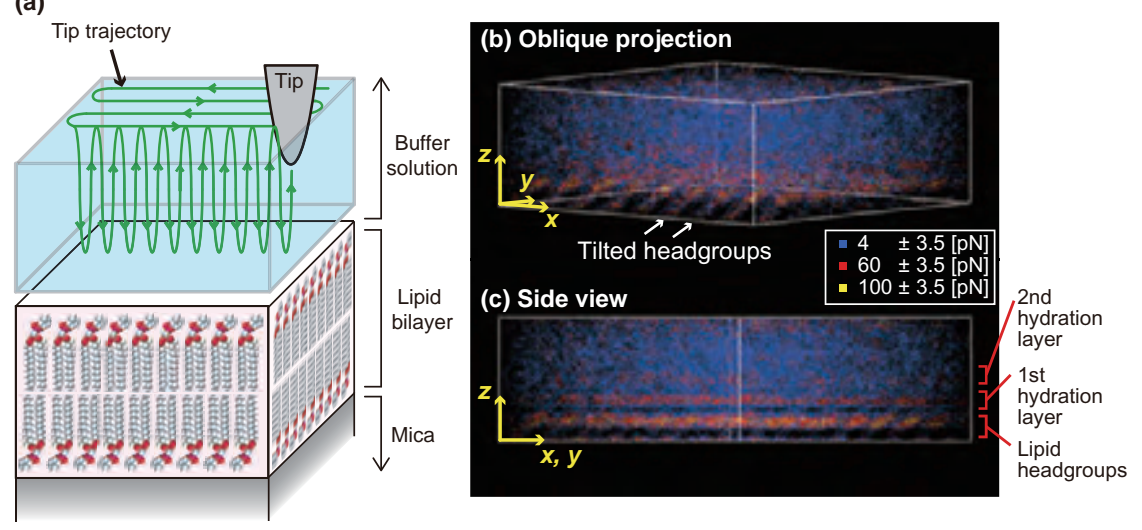

Fig. 10 (a) Illustration of 3D-SFM imaging at an interface between a DPPC bilayer and HEPES buffer solution. (b, c) Scatter plots of the 3D short-range force image of the lipid-water interface. (Reused with permission from Asakawa et al [26]. Copyright 2012, American Chemical Society.)

There are many other examples where local interaction between fluctuating structures and water influences structure and dynamics of biological systems. However, the information obtained by the conventional techniques is not necessarily sufficient to achieve molecular-scale understanding of such interfacial phenomena. The results obtained in this study suggest that 3D-SFM can complement the missing piece of information. This unique capability should contribute to the progress in the molecular-scale understanding of the various phenomena at biological interfaces.

\section{Summary}

In this chapter, we presented the advanced instrumentation and applications of liquid-environment FM-AFM. For instrumentation, we presented the development of 3D-SFM and the improvements of the fundamental performance of FM-AFM such as force sensitivity and operation speed. 3D-SFM allows us to visualize a 3D force distribution at a solid-liquid interface in less than 1 min with subnanometer resolution. The used of small cantilever gives a seven-fold improvement in the force resolution or a 50 times improvement in the operation speed. By developing a high-speed phase detector, we enabled an atomic-resolution imaging at $1 \mathrm{sec} /$ frame. These instrumentation works have significantly broaden the application range of liquid-environment FM-AFM.

For applications, we showed recent examples of 2D and 3D imaging studies. Compared with the 2D imaging studies reported in the early stage of the liquidenvironment FM-AFM development, the applications are more focused on the understanding of important issues in the individual research field. In the 3D imaging studies, we presented two unique capabilities of 3D-SFM. In the imaging of mica- 
water interfaces, we demonstrated the possibility of imaging 3D hydration structures. In the imaging of lipid-water interfaces, we suggested the possibility of direct $3 \mathrm{D}$ visualization of flexible surface structures.

In the next five years, I expect that the $2 \mathrm{D}$ and $3 \mathrm{D}$ imaging techniques will be used for more practical applications in various research fields. For high-speed FMAFM, first applications in physical chemistry and biology will be demonstrated, which should give strong impacts on the researchers in those areas. In the meanwhile, basic studies on the contrast formation mechanism of the 3D force imaging should be performed to understand the correlation between the contrasts and the hydration structures as well as the flexible surface structures. Another important topic will be the development of surface property measurement techniques based on FM-AFM such as distributions of charges, potential, hydrophilicity, viscoelasticity and chemical sensitivity. Most of these instrumentation and application studies have been already started. Therefore, I expect that the rapid progress of the liquidenvironment FM-AFM techniques should continue at least in the next five years.

Acknowledgements This work was supported by KAKENHI (25706023), Japan Society for the Promotion of Science and ACT-C, Japan Science and Technology Agency.

\section{References}

1. T.R. Albrecht, P. Grütter, D. Horne, D. Ruger, J. Appl. Phys. 69, 668 (1991)

2. F.J. Giessibl, Science 267, 68 (1995)

3. S. Morita, R. Wiesendanger, E. Meyer (eds.), Noncontact Atomic Force Microscopy (Nanoscience and Technology) (Springer Verlag, 2002)

4. S.P. Jarvis, T. Uchihashi, T. Ishida, H. Tokumoto, Y. Nakayama, J. Phys. Chem. B 104, 6091 (2000)

5. S.P. Jarvis, T. Ishida, T. Uchihashi, Y. Nakayama, H. Tokumoto, Appl. Phys. A 72, S129 (2001)

6. K. Kobayashi, H. Yamada, K. Matsushige, Appl. Surf. Sci. 188, 430 (2002)

7. T. Okajima, H. Sekiguchi, H. Arakawa, A. Ikai, Appl. Surf. Sci. 68, 210 (2003)

8. H. Sekiguchi, T. Okajima, H. Arakawa, S. Maeda, A. Takashima, A. Ikai, Appl. Surf. Sci. 61, 210 (2003)

9. T. Uchihashi, M.J. Higgins, Y. Nakayama, J.E. Sader, S.P. Jarvis, Nanotechnology 16, S49 (2005)

10. M.J. Higgins, C.K. Riener, T. Uchihashi, J.E. Sader, R. McKendry, S.P. Jarvis, Nanotechnology 16, S85 (2005)

11. A. Sasahara, S. Kitamura, H. Uetsuka, H. Onishi, J. Phys. Chem. B 108, 15735 (2004)

12. T. Fukuma, K. Kobayashi, K. Matsushige, H. Yamada, Appl. Phys. Lett. 87, 034101 (2005)

13. T. Fukuma, M. Kimura, K. Kobayashi, K. Matsushige, H. Yamada, Rev. Sci. Instrum. 76, 053704 (2005)

14. B.W. Hoogenboom, H.J. Hug, Y. Pellmont, S. Martin, P.L.T.M. Frederix, D. Fotiadis, A. Engel, Appl. Phys. Lett. 88, 193109 (2006)

15. S. Nishida, D. Kobayashi, T. Sakurada, T. Nakazawa, Y. Hoshi, H. Kawakatsu, Rev. Sci. Instrum. 79, 123703 (2008)

16. S. Rode, N. Oyabu, K. Kobayashi, H. Yamada, A. Kühnle, Langmuir 25, 2850 (2009)

17. M. Higgins, M. Polcik, T. Fukuma, J. Sader, Y. Nakayama, S.P. Jarvis, Biophys. J. 91, 2532 (2006) 
18. T. Fukuma, M.J. Higgins, S.P. Jarvis, Biophys. J. 92, 3603 (2007)

19. T. Fukuma, M.J. Higgins, S.P. Jarvis, Phys. Rev. Lett. 98, 106101 (2007)

20. T. Fukuma, A.S. Mostaert, S.P. Jarvis, Nanotechnology 19, 384010 (2008)

21. H. Yamada, K. Kobayashi, T. Fukuma, Y. Hirata, T. Kajita, K. Matsushige, Appl. Phys. Express 2, 095007 (2009)

22. H. Asakawa, T. Fukuma, Nanotechnology 20, 264008 (2009)

23. T. Fukuma, Y. Ueda, S. Yoshioka, H. Asakawa, Phys. Rev. Lett. 104, 016101 (2010)

24. J.E. Sader, S.P. Jarvis, Appl. Phys. Lett. 84, 1801 (2004)

25. N. Kobayashi, S. Itakura, H. Asakawa, T. Fukuma, J. Phys. Chem. C 117, 24388 (2013)

26. H. Asakawa, S. Yoshioka, K. Nishimura, T. Fukuma, ACS NANO 6, 9013 (2012)

27. K. Kimura, S. Ido, N. Oyabu, K. Kobayashi, Y. Hirata, T. Imai, H. Yamada, J. Chem. Phys. 132, 194705 (2010)

28. T. Hiasa, K. Kimura, H. Onishi, M. Ohta, K. Watanabe, R. Kokawa, N. Oyabu, K. Kobayashi, H. Yamada, J. Phys. Chem. C 114, 21423 (2010)

29. T. Hiasa, K. Kimura, H. Onishi, Phys. Chem. Chem. Phys. 14, 8419 (2012)

30. T. Hiasa, K. Kimura, H. Onishi, Coll. Surf. A 396, 203 (2012)

31. T. Hiasa, K. Kimura, H. Onishi, J. Phys. Chem. C 116, 26475 (2012)

32. R. Nishioka, T. Hiasa, K. Kimura, H. Onishi, J. Phys. Chem. C 117, 2939 (2013)

33. T. Sugihara, I. Hayashi, H. Onishi, K. Kimura, A. Tamura, Chem. Phys. 419, 74 (2013)

34. T. Hiasa, H. Onishi, Langmuir 29, 5801 (2013)

35. T. Hiasa, K. Kenjiro, H. Onishi, J. Phys. Chem. C 117, 5730 (2013)

36. M. Watkins, A.L. Shluger, Phys. Rev. Lett. 105, 196101 (2010)

37. M. Harada, M. Tsukada, Phys. Rev. B 80, 035414 (2010)

38. M. Tsukada, N. Watanabe, M. Harada, K. Tagami, J. Vac. Sci. Technol. B 28, C4C1 (2010)

39. M. Watkins, M.L. Berkowitz, A. Shluger, Phys. Chem. Chem. Phys. 13, 12584 (2011)

40. M. Watkins, B. Reischl, J. Chem. Phys. 138, 154703 (2013)

41. S.P. Jarvis, A. Oral, T.P. Weihs, J.B. Pethica, Rev. Sci. Instrum. 64, 3515 (1993)

42. N. Umeda, S. Ishizaki, H. Uwai, J. Vac. Sci. Technol. B 9, 1318 (1991)

43. Y. Martin, C.C. Williams, H.K. Wickramasinghe, J. Appl. Phys. 61, 4723 (1987)

44. S. Morita, R. Wiesendanger, F.J. Giessibl (eds.), Noncontact Atomic Force Microscopy Volume 2 (Nanoscience and Technology) (Springer Verlag, 2009)

45. H. Hölscher, S.M. Langkat, A. Schwarz, R. Wiesendanger, Appl. Phys. Lett. 81, 4428 (2002)

46. B.J. Albers, T.C. Schwendemann, M.Z. Baykara, N. Pilet, M. Liebmann, E.I. Altman, U.D. Schwarz, Nat. Nanotech. 4, 307 (2009)

47. M.B. Viani, T.E. Schäffer, A. Chand, M. Rief, H.E. Gaub, P.K. Hansma, J. Appl. Phys. 86, 2258 (1999)

48. S. Hosaka, K. Etoh, A. Kikukawa, H. Koyanagi, K. Itoh, Microelectronic Engineering 46, 109 (1999)

49. T.E. Schäffer, P.K. Hansma, J. Appl. Phys. 84, 4661 (1998)

50. T. Fukuma, K. Onishi, N. Kobayashi, A. Matsuki, H. Asakawa, Nanotechnology 23, 135706 (2012)

51. T. Fukuma, Rev. Sci. Instrum. 80, 023707 (2009)

52. T. Fukuma, S.P. Jarvis, Rev. Sci. Instrum. 77, 043701 (2006)

53. K. Miyata, S. Usho, S. Yamada, S. Furuya, K. Yoshida, H. Asakawa, T. Fukuma, Rev. Sci. Instrum. 84, 043705 (2013)

54. Y. Mitani, M. Kubo, K. Muramoto, T. Fukuma, Rev. Sci. Instrum. 80, 083705 (2009)

55. K. Miyata, H. Asakawa, T. Fukuma, Appl. Phys. Lett. 103, 203104 (2013)

56. H. Asakawa, K. Ikegami, M. Setou, N. Watanabe, M. Tsukada, T. Fukuma, Biophys. J. 101, 1270 (2011)

57. J. Hendriksen, A.C. Rowat, E. Brief, Y.W. Hsueh, J.L. Thewalt, M.J. Zuckermann, J.H. Ipsen, Biophys. J. 90, 1639 (2006)

58. T.J. McIntosh, A.D. Magid, S.A. Simon, Biochemistry 28, 17 (1989)

59. C. Mineo, G.L. James, E.J. Smart, R.G.W. Anderson, 271, 11930 (1996)

60. M.R. Vist, J.H. Davis, Biochemistry 29, 451 (1990)

61. A.M. Smondyrev, M.L. Berkowitz, Biophys. J. 771, 2075 (1999) 
62. L. Miao, M. Nielsen, J. Thewalt, J.H. Ipsen, M. Bloom, M.J. Zuckermann, O.G. Mouritsen, Biophys. J. 82, 1429 (2002)

63. E. Nogales, S.G. Wolf, K.H. Downing, Nature 391, 199 (1998)

64. Y. Kimura, N. Kurabe, K. Ikegami, K. Tsutsumi, Y. Konishi, O.I. Kaplan, H. Kunitomo, Y. Iino, O.E. Blacque, M. Setou, 285, 22934 (2010)

65. N. Senguttuvan, M. Aoshima, K. Sumiya, H. Ishibashi, J. Cryst. Growth 280, 462 (2005)

66. I. Nicoara, M. Stef, A. Pruna, J. Cryst. Growth 310, 1470 (2008)

67. S. Wakahara, Y. Furuya, T. Yanagida, Y. Yokota, J. Pejchal, M. Sugiyama, N. Kawaguchi, D. Totsuka, A. Yoshikawa, Opt. Mater. 34, 729 (2012)

68. Y. Zhang, X. Xiang, W.J. Weber, Nucl. Instr. and Meth. 266, 2750 (2008)

69. L.A. Perez, G.H. Nancollas, Coll. Surf. 52, 231 (1991)

70. H. Moller, H.E.L. Madsen, J. Cryst. Growth 71, 673 (1985)

71. T. Aoba, O. Fejerskov, Crit. Rev. Oral Biol. Med. 13, 155 (2002)

72. O. Prymak, V. Sokolova, T. Peitsch, M. Epple, 6, 498 (2006)

73. S.M. Hamza, S.K. Hamdona, J. Phys. Chem. 95, 3149 (1991)

74. Z. Amjad, Langmuir 9, 597 (1993)

75. C.H. de Vreugd, J.H. ter Horst, P.F.M. Durville, G.J. Witkamp, G.M. van Rosmalen, Coll. Surf. A 154, 259 (1999)

76. C.Y. Tai, J. Cryst. Growth 206, 109 (1999)

77. C.Y. Tai, .C. P. C, T.M. Tsao, J. Cryst. Growth 290, 576 (2006)

78. J.J. Eksteen, M. Pelser, M.S. Onyango, L. Lorenzen, C. Aldrich, G.A. Georgalli, Hydrometallurgy 91, 104 (2008)

79. P.E. Hillner, S. Manne, P.K. Hansma, A.J. Gratz, Faraday Discuss. 95, 191 (1993)

80. D. Bosbach, G. Jordan, W. Rammensee, Eur. J. Mineral. 7, 267 (1995)

81. G. Jordan, W. Rammensee, Surf. Sci. 371, 371 (1997)

82. M.H. Jr., A.F. White (eds.), Review in Mineralogy: Mineral Water Interface Geochemicstry (Mineralogical Society of America, 1990)

83. E.S. Boek, P.V. Coveney, N.T. Skipper, J. Am. Chem. Soc. 117, 12608 (1995)

84. S. Karaborni, B. Smit, W. Heidug, J. Urai, E. van Oort, Science 271, 1102 (1996)

85. G.R. Edwards, L.F. Evans, A.F. Zipper, Trans. Faraday Soc. 66, 220 (1970)

86. J.L. Caslavsky, K. Vedam, J. Appl. Phys. 42, 516 (1971)

87. Y. Leng, P.T. Cummings, J. Chem. Phys. 124, 074711 (2006)

88. G. Sposito, R. Prost, Chem. Rev. 82, 553 (1982)

89. M. Odelius, M. Bernasconi, M. Parrinello, Phys. Rev. Lett. 78, 2855 (1997)

90. P.B. Miranda, L. Xu, Y.R. Shen, M. Salmeron, Phys. Rev. Lett. 81, 5876 (1998)

91. R. Bergman, J. Swenson, Nature 403, 283 (2000)

92. J. Swenson, R. Bergman, W. Howells, J. Chem. Phys. 113, 2873 (2000)

93. L. Cheng, P. Fenter, K.L. Nagy, M.L. Schlegel, N.C. Sturchio, Phys. Rev. Lett. 87, 156103 (2001)

94. S.H. Park, G. Sposito, Phys. Rev. Lett. 89, 085501 (2002)

95. J.N. Israelachvili, G.E. Adams, Nature 262, 774 (1976)

96. F.J. Giessibl, S. Hembacher, H. Bielefeldt, J. Mannhart, Science 289, 422 (2000)

97. M. Ge, J.H. Freed, Biophys. J. 85, 4023 (2003)

98. P. Ball, Chem. Rev. 108, 74 (2008)

99. Y. Levy, J.N. Onuchic, Annu. Rev. Biophys. Biomol. Struct. 35, 389 (2006)

100. J.F. Nagle, S. Tristram-Nagle, Biochim. Biophys. Acta 1469, 159 (2000)

101. J. Milhaud, Biochim. Biophys. Acta 1663, 19 (2004)

102. E. Márazková, P. Hobza, M. Bohl, D.R. Gauger, W. Pohle, J. Phys. Chem. 109, 15126 (2005)

103. J. Sýkora, P. Kapusta, V. Fidler, M. Hof, Langmuir 18, 571 (2002)

104. M.L. Berkowitz, D.L. Bostick, S. Pandit, Chem. Rev. 106, 1527 (2006)

105. J. Cheng, S. Pautot, D.A. Weitz, X.S. Xie, Proc. Natl. Acad. Sci. USA 100, 9826 (2003)

106. M. Pasenkiewicz-Gierula, Y. Takaoka, H. Miyagawa, K. Kitamura, A. Kusumi, J. Phys. Chem. A 101, 3677 (1997)

107. J. Fitter, R.E. Lechner, N.A. Dencher, J. Phys. Chem. 103, 8036 (1999)

108. W. Zhao, D.E. Moilanen, E.E. Fenn, M.D. Fayer, Biophys. J. 130, 13927 (2008) 